\title{
A BIM Based Construction Site Layout Planning Framework Considering Actual Travel Paths
}

\author{
J.C.P Cheng ${ }^{a}$ and S.S. Kumar ${ }^{a}$ \\ ${ }^{a}$ Department of Civil and Environmental Engineering, \\ The Hong Kong University of Science and Technology, Hong Kong \\ E-mail: cejcheng@ust.hk, ssk@ust.hk
}

\begin{abstract}
-
Construction site layout planning (CSLP) is recognized as a crucial component of construction management. The objective of CSLP is to determine the best arrangement of temporary facilities on a construction site, which would minimize the transportation distance of site personnel and equipment. It could be achieved by creating dynamic layout models that allow layout planners to cater the changing requirements of the site. However, these models are project specific and require large amount of manual data input by the layout planner. Besides being tedious, this approach is not practical either, since any changes to the design or construction plans would have to be manually updated into the models, resulting in unnecessary work by the layout planner. In this study, we propose an automated framework to create dynamic site layout models leveraging the BIM technology. Using the information in BIM models and construction schedules, a dynamic layout model can be automatically created for facility layout optimization. Furthermore, the actual travel distances among facilities instead of Euclidean distances are considered in our framework when performing the facility layout optimization. $A^{*}$ algorithm and genetic algorithm heuristic method are used. The proposed approach and framework could reflect the actual site situation and facilitate the facility layout planning on construction sites. A case example is presented in this paper to demonstrate the framework and compare its results with those using Euclidean distances
\end{abstract}

Keywords -

Construction site layout planning (CSLP); Building information modelling (BIM); Genetic algorithm (GA); Non-Euclidean distance

\section{Introduction}

The objective of construction site layout planning (CSLP) is to determine the optimal layout of temporary facilities (such as storage areas, fabrication shops, machines, residence facilities and equipment) within the boundaries of a construction site in order to enable the safe and efficient movement of materials, equipment and labour [1]. The CSLP problem can be subdivided into three parts - (1) determining the required size of facilities, (2) identifying at which stage of construction each facility is required, and (3) allocating facilities to different site locations.

Site layout models fall under two broad categories static models and dynamic models. Static models assume a fixed layout for temporary facilities through the entire duration of a project, whereas dynamic models attempt to capture the changing facility requirements during different stages of construction [2]. Dynamic models are better than static models in addressing the requirements of a construction site and recent research mainly focuses on dynamic layout planning [3]. However, modelling the dynamic facility requirements of a construction site is a complicated task that takes a significant amount of time and effort by the layout planner. Current site layout planning tools require a large number of project specific variables to be inputted manually by the layout planner. This method is cumbersome, especially since any changes to the original construction plan would require all of the updated project variables to be inputted again.

In this study we demonstrate how construction site layout planning can be automated using building information modeling (BIM) technology. Although BIM has been used in the construction industry for over a decade, its use in construction planning is still limited to clash detection and 4D simulation [4]. However, BIM models are rich information sources and hence can be used for site layout planning as well. Information stored in BIM models can be used as a basis for estimating the size, type and number of temporary facilities required by a construction project over time. This paper will demonstrate how BIM can be used in conjunction with project schedules to facilitate the creation of dynamic layout models for CSLP. Furthermore, since this methodology pivots on BIM, design and construction changes will automatically be reflected in the layout models, significantly reducing unnecessary work by the 
layout planner. This paper presents a CSLP system framework based on BIM that we developed with the consideration of non-Euclidean distance among facilities on construction sites.

An important step of the CSLP process is optimizing the layout of facilities by allocating spaces to facilities so as to ensure efficient site operations. This problem is considered to be 'NP-hard' and several research studies have attempted to arrive at solutions using heuristics [5] and mathematical optimization techniques [6]. In most layout optimization approaches, the goal is to minimize the total inter-facility transportation cost, which can be expressed as:

$$
\operatorname{Min} \sum_{i=1}^{n-1} \sum_{j=i+1}^{n} d_{i j} f_{i j} R_{i j}
$$

where $n$ is the total number of facilities, $f_{i j}$ is the frequency of transportation between facilities $i$ and $j, R_{i j}$ is the unit cost of transportation between facilities $i$ and $j$, and $d_{i j}$ represents the distance travelled by site personnel between facilities $i$ and $j$. Most of the previous studies take the parameter $d_{i j}$, to be the Euclidean or straight line distance. However on a construction site, due to the presence of obstacles and other restrictions, it is impossible for site personnel to always follow straight line paths. Instead they travel from one point to the other avoiding any obstacles that may come in their way. Sanad et al. [7] claimed that using linear distances, such as the Euclidean distances, leads to an under-estimation of the site layout problem and hence introduced the concept of using actual travel paths instead. Yahya and Saka [8] introduced the concept of obstruction distance which was added to the straight line distance to account for site obstacles. However, neither of these studies laid much emphasis on accurately modelling the paths followed by site personnel. In this study, we used the $\mathrm{A}^{*}$ algorithm in order to realistically model the travel patterns of personnel on a construction site. By doing so we could to develop a more accurate estimate of their travel distances thereby improving the reliability of the optimization model. The superiority of the actual travel path driven optimization is demonstrated on an example project.

\section{The BIM Based Construction Site Layout Planning Framework}

In CSLP, construction facilities can be categorized as either fixed facilities or temporary facilities. Fixed facilities, such as buildings under construction, are assumed to be immovable and their locations are fixed prior to construction. Temporary facilities, on the other hand, refer to site objects that do not have fixed locations prior to construction and need to be allotted space on the construction site through careful planning. Temporary facilities may refer to laydown areas, fabrication shops, site offices, batch plants, etc. Since the site layout represents the work conditions of the labour crew for the entire duration of construction, thorough consideration must be put into deciding the site layout. Proper layout planning ensures good working conditions and hence improves the morale and hence productivity of the entire labour force. The CSLP process can be subdivided into three major tasks: (1) estimating size requirements of facilities, (2) identifying at which stage of construction each facility is required, and (3) allocating each facility a position on the construction site. The following subsections will describe these three tasks and how BIM can be leveraged to perform these tasks.

\subsection{Facility Sizing}

Facilities should be sized so as to facilitate (1) best practices for material storage, (2) safe work conditions for labourers, and (3) efficient functioning of the facility. Material storage facilities should ensure that there is adequate space for storage throughout the duration of construction. Facilities such as fabrication shops, carpentry yards and site offices should provide an unhindered working environment. Production facilities such as batching plants must be sized keeping in mind their production capacities. Therefore, the size of temporary facilities depend upon variables such as the estimated quantity of work, rate of consumption of resources, number of workers and site area. Using information gleaned from prior projects, layout planners generally develop thumb rules or parametric

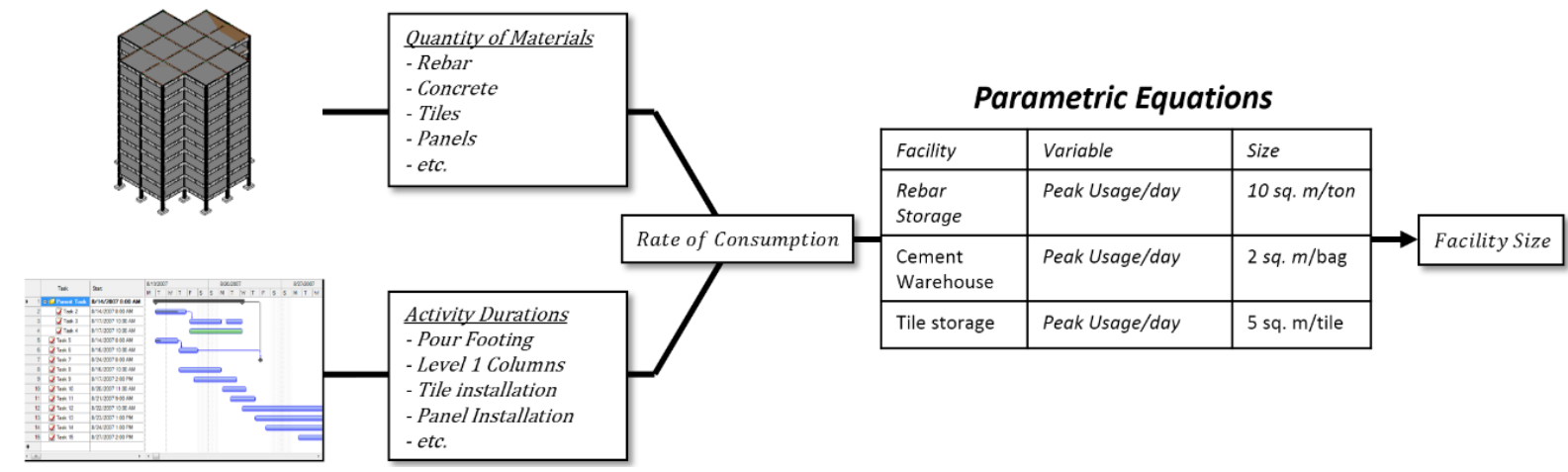

Figure 1. Calculating facility sizes 
relationships to estimate facility size requirements. Based on interviews with practitioners, we developed a set of relationships between facility sizes and their dependent variables, as shown in Table 1. In general, the facility sizes depend on the peak consumption rates of resources.

Table 1. Parametric relationships for facility sizes

\begin{tabular}{lll}
\hline Facility & Dependent Variable & Size \\
\hline Rebar & Peak consumption / day & $10 \mathrm{~m}^{2} /$ ton \\
Storage & & \\
Rebar \\
$\begin{array}{l}\text { Bending } \\
\text { Tiles }\end{array}$ & Peak consumption/day & $10 \mathrm{~m}^{2} /$ ton \\
$\begin{array}{l}\text { Storage } \\
\text { Cement }\end{array}$ & Peak consumption/day & $5 \mathrm{~m}^{2} / \mathrm{box}$ \\
$\begin{array}{l}\text { Storage } \\
\text { Engineer's } \\
\text { Caravan }\end{array}$ & Total engineering crew & $15 \mathrm{~m}^{2} /$ person \\
\hline
\end{tabular}

The process of facility sizing could be automated by adopting the following steps, as shown in Figure 1:

Step 1: Calculating the total amount of resources required by each activity. By performing quantity takeoffs on the BIM model, we were able to obtain detailed information pertaining to the quantity of resources consumed by each activity. Thus, for example, the total volume of rebar and concrete needed for an activity such as "Level 1 Columns" could be derived directly from the BIM model.

Step 2: Calculating the durations of activities. This information could be read from the planned construction schedule. For example, the duration of activity "Level 1 Columns" would refer to the number of days assigned to this activity in the project schedule.

Step 3: Determining the peak rate of consumption. For each activity, the rate of consumption was calculated by dividing the quantity of resources consumed by the duration of the activity. The peak consumption rate could then be obtained as the maximum rate of consumption among all activities.

$$
\begin{aligned}
& \text { Peak rate of consumption } \\
& =\text { Max }\left(\frac{\text { quantity consumed }}{\text { duration of consumption }}\right)
\end{aligned}
$$

Step 4: Applying the parametric relationship to yield the required facility size. For example, if the peak rate of rebar consumption is 7.85 ton/day, the required size of the rebar storage yard could be calculated as $78.5 \mathrm{~m}^{2}$ by multiplying the peak rate of consumption by the parametric relationships. The size of the engineer's caravan could be computed assuming six engineers.

In this work, Autodesk Revit was used as the BIM tool and Microsoft Project was used for scheduling. The planned construction schedule as well as the resource information from the BIM model were exported into Excel spreadsheets. We then created a program to read these files, perform the necessary calculations and hence obtain the required facility sizes (see Figure 1). Changes to the design and schedule could be updated into the Excel spreadsheets, enabling them to be reflected in the final calculations. Therefore, our framework allows a quick and easy method for facility sizing and does not require the users to manually input project specific information. This framework also achieves a significant reduction in effort when coping with changes to design and construction plans.

\subsection{Dynamic Layout}

Not all facilities are required throughout the whole project from start to finish. Many facilities are required on site only for limited durations after which they are dismantled. The space which they occupied then becomes available for setting up other facilities. On sites with limited available space, multiple facilities might occupy the same position on the site during different stages of construction. In order to model these changing space requirements, the CSLP process must be treated as a dynamic layout optimization problem. Dynamic layout refers to the sequence of layouts spread over distinct time intervals, which when taken together span the entire duration of construction [9]. In other words, the construction project is split into multiple phases and layouts for each phase have to be determined individually. Tommelein et al [9] developed a dynamic layout tool called MovePlan which took activity relationships as input in order to determine the dynamic facility requirements of a site. In this study we have developed a methodology of determining the dynamic layout requirements from the planned construction schedule. This was done by mapping each activity on the schedule to its corresponding facility requirements, as depicted in Figure 2.

For instance, in order to determine during which time interval of construction a rebar storage yard would be needed, we would first need to identify (1) the activities which require a rebar storage yard, and (2) their start and end dates. Using the logic that a facility should be present on site only as long as there is an activity which requires it, we could correlate the requirement of the rebar storage yard to the durations of related activities.

Most commercial software allows for schedules to be exported as csv files, enabling us to create a program to read activities and assign them to phases. As a result, the process of dynamic modelling could be automated. 


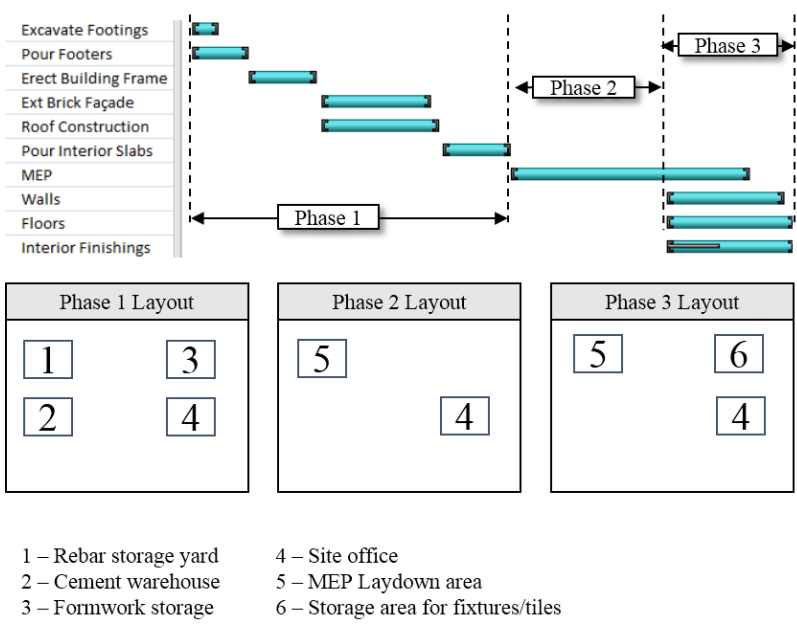

Figure 2. Relationship between schedule and layout phases.

\subsection{Objective Function of the Facility Layout Optimization Problem}

After identifying the size and number of facilities required in each phase of construction, we need to formulate the CSLP problem into an optimization problem. On a construction site, personnel travel from one facility to the other in order to perform activities or transport materials. Every such movement is assumed to incur a cost which is directly proportional to the distance travelled. The objective of layout planning is to minimize the total inter-facility transportation cost of materials and labour while adhering to a specified level of safety. This is aligned with the principles of lean construction, which recommend minimizing unnecessary material handling and transportation costs. The objective function was mathematically defined as:

$$
\operatorname{Min} \sum_{p=1}^{N} \sum_{i=1}^{n-1} \sum_{j=i+1}^{n} d_{i j p} R_{i j p} f_{i j p} P_{i j p}
$$

s.t.

$$
\begin{gathered}
\sum_{p=1}^{N} \sum_{i=1}^{n-1} \sum_{j=i+1}^{n} e_{i j p} P_{i j p} \leq S_{i j p} \\
e_{i j}=\sqrt{\left(x_{i}-x_{j}\right)^{2}+\left(y_{i}-y_{j}\right)^{2}}
\end{gathered}
$$

where $\left(x_{i}, y_{i}\right)$ and $\left(x_{j}, y_{j}\right)$ are the Cartesian coordinates of the centroids of facilities $i$ and $j$ respectively, $N$ refers to the number of phases, $n$ refers to the total number of facilities, $d_{i j p}$ refers to the actual travel distance between facilities $i$ and $j$ during phase $p$ considering the presence of site obstructions, which will be explained in the next section. $R_{i j p}$ and $f_{i j p}$ refer to the cost and frequency of transportation between facilities $i$ and $j$ during phase $p . P_{i j p}$ is a parameter which denotes the presence of facilities $i$ and $j$ during phase $p$. Eq. (3) represents the total distance travelled by site personnel during construction. Having facilities too close to one another creates a safety problem, especially when heavy materials are being lifted by cranes. This is expressed in Eq. (4), where $S_{i j p}$ denotes the trade-off between safety and closeness between two facilities.

The layout corresponding to the minimum total travel distance is assumed to represent the best possible layout.

\subsection{Actual Travel Distance}

A drawback of most studies on this topic is that they used linear distances in facility layout optimization. Park et al. [10] demonstrated the superiority of using actual travel paths over linear distances in solving the floor-level material layout problem for an indoor environment. In this study we have used the actual travel distances of site personnel instead of unrealistic linear distances. It is a known fact that on a construction site, personnel always seek the shortest path from source to destination in a bid to minimize their effort in transportation. Hence, the actual travel path is assumed to be the shortest path from one facility to another, considering the presence of site obstacles.

To determine the actual travel path, we converted the site into a grid, mapped the locations of obstacles on it, and then used the $\mathrm{A}^{*}$ algorithm to compute the shortest path between two points, as illustrated in Figure 3. In order to ease the computation effort we limited the path to move in eight independent directions. This leads to a slight deviation in the actual travel path of personnel and the path generated by the $\mathrm{A}^{*}$ algorithm. However, this does not take away from the fact that the distance calculated by the $\mathrm{A}^{*}$ algorithm is more accurate than the Euclidean distance.

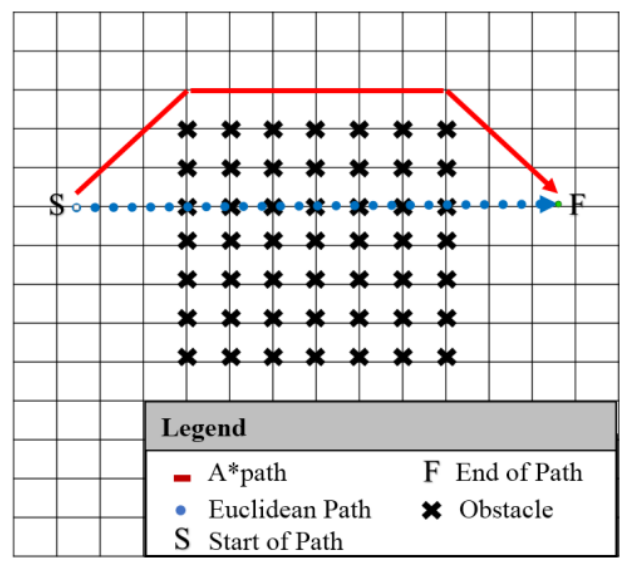

Figure 3. Linear and Actual Travel Paths 
Another characteristic of construction sites is the width of the path required by personnel and equipment. Light materials may be carried by labourers whereas heavier materials require wheelbarrows or forklifts for transportation. The width of the path required is different in each case and is related to the size of the equipment. In order to ensure that different locations are accessible by both labour and equipment, it is essential to select layouts which provide safe and navigable paths In our model we assumed the path width as $1 \mathrm{~m}$ for labourers, $2 \mathrm{~m}$ for wheelbarrows and $5 \mathrm{~m}$ for larger equipment. This allows a more accurate representation of site activities.

\subsection{Constraints of the Facility Layout Optimization Problem}

\subsubsection{Available Site Space}

$\mathrm{BIM}$ encompasses all of the functionalities of CAD, hence by extracting the site layout plans of the construction project, we were able to automatically generate available workspaces. This was done by extracting data contained in the layout plans and formulating them into a set of mathematical inequalities. The closed area bounded by the inequalities represented the total site area available for construction. The fixed facilities (buildings) were then identified in a similar manner and subtracted from the total area encompassed by the site. The remaining area represents the total space allocated for setting up of temporary facilities.

\subsubsection{Overlapping Constraint}

We used a mathematical inequality to ensure that facilities do not overlap with one another or the building to be constructed. Representing a facility by the coordinates of one corner $(x, y)$ the constraint to avoid overlapping between facilities $i$ and $j$ can be expressed as:

$$
\begin{array}{r}
\max \left[\left(x_{j}-x_{i}-l_{i}\right)\left(x_{j}-x_{i}+l_{j}\right),\left(y_{j}-y_{i}\right.\right. \\
\left.\left.-w_{i}\right)\left(y_{j}-y_{i}+w_{i}\right)\right] \geq 0
\end{array}
$$

where $l$ and $w$ represent the length and width of the facility respectively.

\subsubsection{Tower Crane Constraint}

Some facilities such as material storage yards need to be located within the reachable radius of the tower crane. This is mathematically represented as:

$$
\left(x_{t}-x_{f}\right)^{2}+\left(y_{t}-y_{f}\right)^{2} \leq R_{t}^{2}
$$

where $\left(x_{t}, y_{t}\right)$ represents the axis of rotation of the tower crane, $\left(x_{f}, y_{f}\right)$ represents the corner of the facility which is farthest away from the tower crane and $R_{t}$ represents the tower crane's pickup radius.

\subsubsection{Site Accessibility Constraints}

In order to facilitate smooth site operations, facilities should not obstruct the paths of material delivery trucks or other machines used on site. For the safety of the site personnel it is also necessary to ensure that work areas are not too close to these paths. These requirements could be incorporated into the model by defining the commonly used paths by trucks and machines and maintaining a clear distance between facilities and these paths.

\subsubsection{Miscellaneous Constraints}

Based on the layout engineer's discretion and environmental concerns, a range of project specific constraints may be added. Sometimes the site may share one of its boundaries with a school or hospital. In such situations it must be ensured that facilities which generate a lot of noise should not be positioned near this boundary. In certain situations from prior experience, the layout engineer might require a certain relationship in the locations of two facilities. Such conditions can be formulated into mathematical relationships and incorporated into the optimization model.

\subsection{Optimization using Genetic Algorithm}

The optimization problem was solved using Genetic Algorithms (GA), which is a popular heuristic for solving the site layout problem. First a randomly generated large set of initial layouts is populated. The size, shape, orientation and coordinates of each facility were then encoded into genes and measured against a fitness function. The fitness function was taken as the inverse of the objective function. Thus, a layout with a large total travel distance will have a lower fitness value than a layout with a smaller total travel distance. We used the technique of Best First Search (BFS) in order to reduce the convergence time of the GA. The next step involves mimicking the process of natural selection by selecting the fittest genes, allowing them to create offspring and eliminating weaker genes. Each offspring undergoes a crossover, mutation and inversion operation with a certain probability, to prevent them from getting stuck at a local minimum value. The process is repeated over and over again until the genes in successive generations show no significant improvement. Finally, the gene with the best fitness is assumed to represent the solution of the optimization problem. 


\section{Demonstrative Example}

The developed BIM based CSLP framework was tested and illustrated using an example project as follows. The project involves construction of a multistorey reinforced concrete commercial building and an adjacent site office on a construction site of area $3600 \mathrm{~m}^{2}$. Autodesk Revit was used to create the BIM model with all of the material and structural information, as shown in Figure 4. The schedule was created using Microsoft Project allocating 300 days for the completion of construction activities.

By exporting the site layout plan from Revit, we could identify the available workspace as well as the boundary conditions of the site. The detected space was discretized into a square grid of 60 units x 60 units, to facilitate the use of the $\mathrm{A}^{*}$ algorithm. Using the quantity take-off option in Revit 2014, we were able to export material information from the BIM model into csv files. Using the previously defined methodology, we were then able to determine the size of facilities such as the (1) scaffolding stockpile, (2) cement warehouse, (3) rebar storage yard, (4) rebar bending yard, (5) storage for mechanical fixtures, (6) storage for tiles, and (7) storage for panels. The size of the engineer's caravan was selected to be $80 \mathrm{~m}^{2}$ based on the assumption that totally 12 engineers would be needed for the project. It was assumed that labourers would make multiple daily trips from temporary facilities to the required work locations inside the buildings. For activities involving rebar, we assumed that labour personnel would travel from the rebar storage yard to the rebar bending yard, and from the rebar bending yard to their respective work locations. For the sake of simplicity we assumed the centroids of the facilities to be the starting and ending points of each path. The construction schedule on Microsoft Project was exported as a csv file into our program which subsequently generated the facility requirements pertaining to three phases of construction. The required facility sizes and the phase of construction in which they appear are shown in Table 2.

Once the facility requirements were determined, we moved on to optimizing their layouts. A randomly generated set of 2000 layouts was initially populated and tested against the fitness function. We selected the top 500 layouts, with respect to their fitness values, as our initial population and proceeded with optimization using GA.

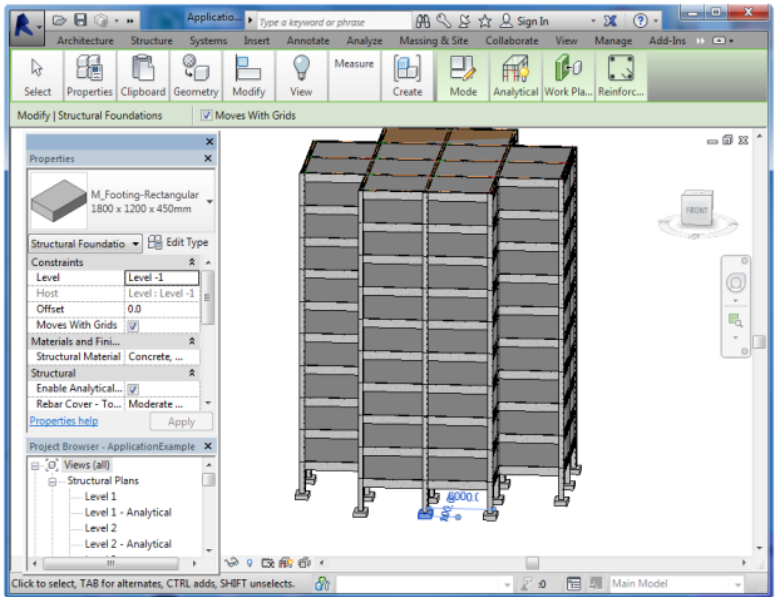

Figure 4. Revit model of the building

Table 2. Temporary Facilities, their size and phase of requirement

\begin{tabular}{cccc}
\hline No. & Temporary Facility & $\begin{array}{c}\text { Size } \\
\left(\mathrm{m}^{2}\right)\end{array}$ & Phase \\
\hline F1 & Engineer's caravan & 80 & P1,P2,P3 \\
F2 & Scaffolding & 65 & P1,P2 \\
& stockpile & & \\
F3 & Cement warehouse & 75 & P1,P2 \\
F4 & Rebar storage yard & 80 & P1 \\
F5 & Rebar bending yard & 80 & P1 \\
F6 & MEP storage & 75 & P2,P3 \\
F7 & Tiles storage & 80 & P3 \\
F8 & Storage for panels & 85 & P3 \\
T.C. & Tower Crane & 25 & P1,P2,P3 \\
\hline
\end{tabular}

\subsection{Results}

Table 3 compares the layouts generated from two cases: (1) Euclidean distance (the conventional method) and (2) actual travel distance (the proposed method). The layout generated from the conventional method yielded a total Euclidean distance of $2,700 \mathrm{~km}$, whereas the actual travel distance of this layout was calculated to be $3,750 \mathrm{~km}$. This shows that the presence of obstacles on the site accounted for the actual travel distance to be significantly greater than the centre to centre distance. This finding strongly highlights the under-estimation of the site layout problem by using linear distances. Figure 5 shows the layouts generated by the two approaches.

The actual travel distance driven optimization, yielded in a total travel distance of $3,130 \mathrm{~km}$, a reduction of $16.5 \%$ over the linear method. This shows that linear distance based optimization could lead to the generation of sub-optimal layouts. Furthermore, with an increase in the number of obstacles on the site, the superiority of the actual travel distance driven optimization becomes 
even more significant. These results confirm that, optimizing the actual travel distance between facilities, generates layouts which significantly reduce unnecessary site-level transportation.

Table 3. Comparison of layouts using linear distances and actual travel paths

\begin{tabular}{ccc}
\hline & $\begin{array}{c}\text { Total } \\
\text { Euclidean } \\
\text { distance }(\mathrm{km})\end{array}$ & $\begin{array}{c}\text { Total actual } \\
\text { travel distance } \\
(\mathrm{km})\end{array}$ \\
\hline $\begin{array}{c}\text { Layout obtained } \\
\text { considering linear } \\
\text { Euclidean paths }\end{array}$ & 2,700 & 3,750 \\
\hline $\begin{array}{c}\text { Layout obtained } \\
\text { considering actual } \\
\text { travel paths }\end{array}$ & 2,900 & 3,130 \\
\hline
\end{tabular}

\section{Conclusion}

This paper presents a methodology framework which enables automating the CSLP process by leveraging the BIM technology. Based on this methodology framework, we created a tool to optimize the dynamic layouts of facilities on a construction site. This tool is flexible to changes in the design or construction plans and would significantly decrease the time spent in layout planning. Furthermore, by considering the actual travel distances in the CSLP optimization problem, a more accurate representation of construction activities is achieved. In the demonstrative example, our method generated layouts which reduced the total travel distance by $16.5 \%$, a significant improvement over conventional methods. This improvement would be even more pronounced when dealing with larger sites with more obstacles. Future efforts will aim to integrate this model with 4D construction simulation to further aid the decision making of layout planners.

\section{References}

[1] Harmanani, Zouein and Hajar, "Genetic Algorithm for Solving Site Layout Problem with UnequalSize and Constrained Facilities," Journal of Computing in Civil Engineering, 16(2):143-151, 2002.

[2] Ning, Lam and Lam, "Dynamic construction site layout planning using max-min ant system," Automation in construction, 19(1):55-65, 2010.

[3] Xu and Li, "Multi-objective dynamic construction site layout planning in fuzzy random environment," Automation in Construction, 27:155-169, 2012.

[4] Azhar, "Building information modeling (BIM): Trends, benefits, risks, and challenges for the AEC industry," Leadership and Management in Engineering, 11(3):241-252, 2011

[5] Li and Love, "Site-Level Facilities Layout Using Genetic Algorithms," Journal of Computing in Civil Engineering, 2(4):227-231, 1998.

[6] Easa, Said and Hossain, "New mathematical optimization model for construction site layout," Journal of Construction Engineering and Management, 134(8):653-662, 2008.
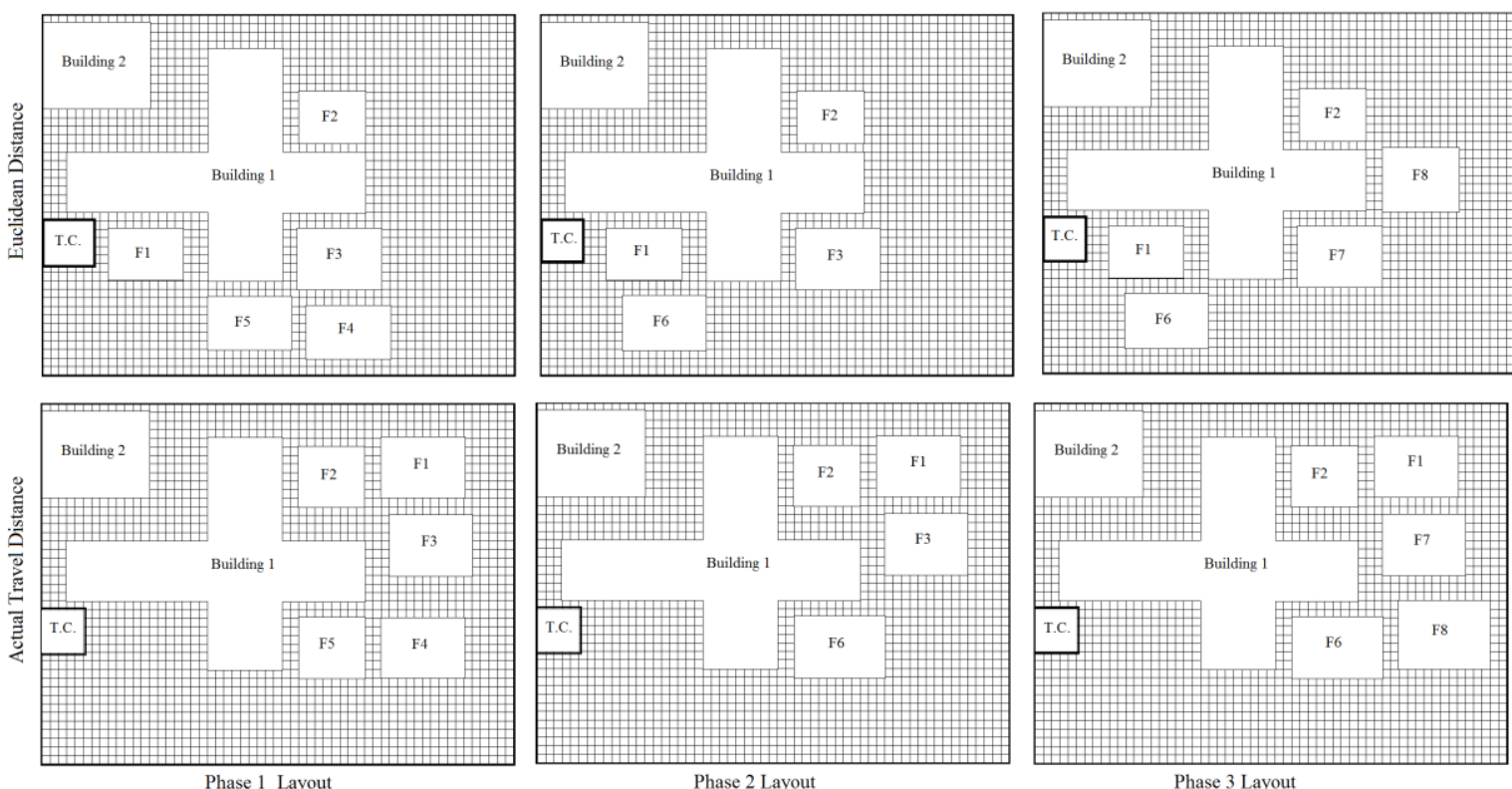

Figure 5. Comparison of layouts from linear distance and actual travel distance driven optimization 
[7] Sanad, Ammar and Ibrahim, "Optimal Construction Site Layout Considering Safety and Environmental Aspects," Journal of Construction Engineering and Management, 134(7):536-544, 2008.

[8] Yahya and Saka, "Construction site layout planning using multi-objective artificial bee colony algorithm with Levy flights," Automation in Construction , 38:14-29, 2014.

[9] Tommelein and Zouein, "Interactive Dynamic Layout Planning," Journal of Construction Engineering and Management, 119(2):266-287, 1993.

[10] Park, Yang, Lee, Han and Ji, "Floor-Level Construction Material Layout Planning Model Considering Actual Travel Path," Journal of Construction Engineering and Management, 138(7):905-915, 2012. 